\title{
Calculation of linear ship waves
}

\author{
D. C. Scullen ${ }^{1}$
}

(Received 31 January 2010; revised 10 May 2010)

\begin{abstract}
The Sea Wave Pattern Evaluation suite of programs was originally developed to calculate far field wave elevations for thin ships but grew to encompass a raft of other tasks including calculating the complete wave field with velocity components for multihull and submerged vessels, incorporating sinkage and trim, viscous damping and finite depth effects, and measuring wave resistance and viscous resistance. It also led to investigations into waves produced by pressure distributions and planing surfaces. This article traces Sea Wave Pattern Evaluation's development, explains the mechanics of its key components, and refers to some of the results that have been produced by it.
\end{abstract}

\section{Contents}

1 Introduction

C317

2 Mathematical formulation

C317

http://anziamj . austms.org.au/ojs/index.php/ANZIAMJ/article/view/2885 gives this article, (c) Austral. Mathematical Soc. 2010. Published June 20, 2010. ISSN 1446-8735. (Print two pages per sheet of paper.) 
4 Conclusion

C329

References

C330

\section{Introduction}

SwPE calculates the free surface elevations and fluid particle velocities about ships and submarines subject to the assumptions of thin-ship theory. Its major advantage is its speed of execution which allows the free surface to be evaluated at high resolution (of the order of 100,000 field points) in a matter of minutes.

The program was developed by the author jointly with E. O. Tuck and L. Lazauskas between 1999 and 2004, during which time a series of reports $[5,6,7,8,9,10,11,12]$ was prepared documenting each stage of development. Several years have passed and it is interesting to reflect on the project as a whole. This document summarises the major components of SWPE in the order that they were developed.

\section{Mathematical formulation}

We adopt a coordinate system in the frame of reference of the vessel where $x$ increases from bow to stern, $y$ increases to starboard and $z$ increases vertically upwards. Assuming steady and irrotational flow of an inviscid and incompressible fluid allows the simplification to potential flow theory whereby the velocity potential $\Phi(x, y, z)$ satisfies Laplace's equation $\nabla^{2} \Phi=0$ and the fluid particles' velocities are prescribed by $\mathbf{q}=\nabla \Phi$.

In general, the free surface boundary conditions are the dynamic boundary 
condition that the pressure $p$ is a constant (arbitrarily zero) on the free surface $z=Z(x, y)$, and the kinematic boundary condition that there be no flow through the free surface, that is $\mathbf{q} \cdot \mathbf{n}=0$.

Defining the perturbation to the potential of a free stream with velocity $\mathrm{U}$ as $\phi=\Phi-\mathrm{U} x$ and substituting into Bernoulli's equation for pressure $\mathrm{p} / \rho=$ $\left[\mathrm{U}^{2}-(\nabla \Phi)^{2}\right] / 2-\mathrm{gz}$ and then neglecting terms of order $\mathcal{O}\left(\phi^{2}\right)$ yields a linearised dynamic boundary condition from which the free surface elevation can be calculated as $\mathbf{Z}=-\mathrm{U} \phi_{x}(x, y, 0) / g$. Undertaking a similar procedure for the kinematic boundary condition yields the linearised kinematic or Kelvin free surface boundary condition $\phi_{x x}+k_{0} \phi_{z}=0$ on $z=0$ where $k_{0}=g / u^{2}$.

Havelock sources satisfy both Laplace's equation and the Kelvin condition and therefore present a particular solution for the linearised problem. One form for the velocity potential of a Havelock source of unit strength located at depth $|\zeta|$ beneath the origin is [14]

$$
\begin{aligned}
G(x, y, z ; \zeta)= & -\frac{1}{4 \pi}\left(\frac{1}{R}-\frac{1}{R_{0}}\right)+\frac{k_{0}}{2 \pi^{2}} \mathfrak{R} \int_{-\pi / 2}^{\pi / 2} d \theta \sec ^{2} \theta \\
& \int_{0}^{\infty} d k e^{-i k(x \cos \theta+y \sin \theta)+k(z+\zeta)} \frac{1}{k-k_{0} \sec ^{2} \theta}
\end{aligned}
$$

where $R$ is the distance between the point of evaluation and the source and $R_{0}$ is the same but for the image of the source, reflected in the plane $z=0$. The path of integration passes above the pole so that the waves lie aft of the source. If the path of integration passes beneath the pole then the solution is reflected in the plane $x=0$ and the waves lie ahead of the source.

The vessel's hull $y=\eta \pm Y(x, z)$ is subject to the Neumann boundary condition $\mathbf{q} \cdot \mathbf{n}=0$ which specifies that flow is tangential to the hull's surface. Its linearised approximation $\phi_{y}=U Y_{x}$ is satisfied by a continuous distribution of Havelock sources over the hull centreplane B with strength $\sigma=2 \mathrm{UY} Y_{x}[1]$. 
Thus, according to thin ship theory, the potential can be calculated as

$$
\phi(x, y, z)=2 \mathrm{U} \iint_{\mathrm{B}} \mathrm{d} \xi \mathrm{d} \zeta Y_{\xi}(\xi, \eta, \zeta) \mathrm{G}(x-\xi, y-\eta, z ; \zeta) .
$$

In terms of computational effort, this is a quadruple integral which is to be evaluated at each point of interest in the fluid.

A further simplification can be made when the Havelock source is decomposed into local and far field components so that $G=G^{L}+G^{F}$. The local field component $\mathrm{G}^{\mathrm{L}}$ is defined by $\mathrm{G}^{\mathrm{L}}(x, y, z ; \zeta)=\mathrm{G}(-|x|, y, z ; \zeta)$. Being identical to $G$ ahead of the source, this function raises the free surface smoothly and monotonically as it approaches the source, and being fore-aft symmetric the free surface then decreases smoothly and monotonically back to $z=0$ after passing the source. Polynomials that approximate $\mathrm{G}^{\mathrm{L}}$ and that are evaluated rapidly have been developed by Newman [2]

The far field component $G^{F}$ is zero ahead of the source. Recalling that the path of integration passing beneath the pole produces a reflected solution and denoting that function as $\mathrm{G}^{-}$we recognise that for $x>0, \mathrm{G}^{\mathrm{F}}=\mathrm{G}-\mathrm{G}^{-}$ and is therefore given by a path of integration that completely circles the pole clockwise. Thus, for $x>0, G^{F}$ is $-2 \pi i$ times the residue at the pole, specifically

$$
\mathrm{G}^{\mathrm{F}}(x, y, z ; \zeta)=-\frac{k_{0}}{\pi} \mathfrak{R i} \int_{-\pi / 2}^{\pi / 2} \mathrm{~d} \theta \sec ^{2} \theta e^{-i k_{1}(x \cos \theta+y \sin \theta)+k_{1}(z+\zeta)}
$$

where $k_{1}=k_{0} \sec ^{2} \theta$. The advantage of this decomposition is that $\mathrm{G}^{\mathrm{F}}$ involves only a single integral, the dependence on $k$ having been eliminated.

Thus, replacing $G$ by $G^{\mathrm{L}}+\mathrm{G}^{\mathrm{F}}$ one decomposes the potential into local and far field components $\phi=\phi^{\mathrm{L}}+\phi^{\mathrm{F}}$ and finds expressions for each. The local field component

$$
\phi^{\mathrm{L}}(x, y, z)=2 \mathrm{U} \iint_{B} \mathrm{~d} \xi \mathrm{d} \zeta Y_{\xi}(\xi, \eta, \zeta) G^{\mathrm{L}}(x-\xi, y-\eta, z ; \zeta)
$$


requires a double integral to be computed at every point of interest in the fluid, with $G^{\mathrm{L}}$ to be evaluated of the order of 1000 times for each.

The far field component is

$$
\phi^{\mathrm{F}}(x, y, z)=2 \mathrm{U} \iint_{B} \mathrm{~d} \xi \mathrm{d} \zeta Y_{\xi}(\xi, \eta, \zeta) G^{\mathrm{F}}(x-\xi, y-\eta, z ; \zeta) .
$$

By swapping the orders of integration to

$$
\begin{aligned}
& \phi^{\mathrm{F}}(x, y, z) \\
& =-2 \mathrm{U} \frac{\mathrm{k}_{0}}{\pi} \mathfrak{R} \int_{-\pi / 2}^{\pi / 2} \mathrm{~d} \theta \sec \theta \mathrm{k}_{1} e^{-i k_{1}(x \cos \theta+y \sin \theta)+k_{1} z}\left[\mathrm{P}_{\mathrm{T}}^{\mathrm{F}}(\theta)+i \mathrm{Q}_{\mathrm{T}}^{\mathrm{F}}(\theta)\right]
\end{aligned}
$$

where the free wave spectrum is

$$
\mathrm{P}_{\mathrm{T}}^{\mathrm{F}}(\theta)+i \mathrm{Q}_{\mathrm{T}}^{\mathrm{F}}(\theta)=\frac{1}{-i k_{1} \cos \theta} \iint_{\mathrm{B}} \mathrm{d} \xi \mathrm{d} \zeta Y_{\xi}(\xi, \eta, \zeta) e^{i k_{1}(\xi \cos \theta+\eta \sin \theta)+k_{1} \zeta}
$$

one moves the dependence on the location of the field point to the outer integral. This reduces its computational workload to performing a double integration over the body only once (for each $\theta$ ), followed by a single integration to be evaluated at every point of interest in the fluid. Typically, of the order of 1000 points are used to represent the hull so applying this technique leads to a reduction in computation time by a factor of 1000. Also, if the original $k$-integration would have been computed using 100 values for $k$ then a further reduction in computation time by a factor of 100 has been achieved. Combined, the far field potential is calculated by as much as 100,000 times faster than could a naïve implementation of (1) for the total potential.

\section{Development of SWPE}

Prior to embarking on SWPE, the team members had developed some interesting programs and experience. Leo Lazauskas, under the guidance of 
Ernie Tuck, had developed programs Michlett and Polymich which calculated wave resistance from the free wave spectrum and had been used for minimising wave resistance of multihulls. Importantly though, neither program was able to compute wave elevations. I had developed the program Nonlinear Free-Surface Flow Solver (NFSFS) [3] which determined nonlinear waves and forces for submerged vessels. Each of those programs and the skills and experience developed with them played an important role in the development of SWPE.

Michlett and Polymich were able to compute wave resistance which is similar in computational complexity to the far field potential. Both require calculation of $\mathrm{P}_{\mathrm{T}}^{\mathrm{F}}+i \mathrm{Q}_{\mathrm{T}}^{\mathrm{F}}$ and each involves a further integration with respect to $\theta$ to be performed, although the integration for the far field component is technically more challenging because its integrand is rapidly oscillating. This led to the first version of SWPE (SWPE v1) which computed far field wave elevations plus surface velocity components aft of the stern. The restriction of the program to a region aft of the stern was based on our mistaken belief that $\mathrm{P}_{\mathrm{T}}^{\mathrm{F}}+i \mathrm{Q}_{\mathrm{T}}^{\mathrm{F}}$ necessarily required the double integration to be performed over the whole of the hull's centreplane. At any rate, the value of the far field component is as an approximation to the total flow, and this approximation is only applicable aft of the stern. A feature of SWPE is that the calculation of the $\mathrm{P}_{\mathrm{T}}^{\mathrm{F}}+i \mathrm{Q}_{\mathrm{T}}^{\mathrm{F}}$ employed Filon quadrature to significantly enhance the accuracy and speed of the computation [5].

SWPE represents the hull as a grid of equally spaced vertical stations and equally spaced horizontal waterlines, with an offset $Y(x, z)$ being specified at each intersection. SwPE v1 was slightly limited in that the highest waterline needed to be located at exactly $\mathrm{z}=0$ so that only the submerged portion of the hull was specified. This precluded the calculation of flows for totally submerged vessels such as submarines unless several waterlines each with all offsets being zero were specified between the top of the hull and the sea's surface. Also, because the waterlines needed to be evenly spaced and the uppermost waterline needed to be at $z=0$, changing the depth of submergence involved supplying a different set of hull offsets. This was both cumbersome 
and computationally wasteful and was easily rectified by replacing $\zeta$ by $\zeta+\mathrm{H}$ in the formulation, where a negative value of $\mathrm{H}$ represented the depth of submergence of the uppermost waterline. For the far field component this was achieved simply by multiplying the integrand of (5) by a factor of $e^{k_{1} \mathrm{H}}$. With this minor change to the program, the second version, SWPE v2, was able to produce flows around submarines of arbitrary depth, without the need to modify the offsets provided.

This allowed comparison between the results produced by SWPE with those produced by NFSFS, which could calculate the nonlinear solution but only for completely submerged bodies. To accommodate this, NFSFS was modified from full nonlinear (Neumann-Stokes) mode to operate in two other modes - one in which the free surface conditions were linearised (NeumannKelvin mode) and another in which the hull was represented by a distribution of sources along the centreplane with strength $\sigma=2 U Y_{x}$ (Michell-Kelvin mode). While the comparison between SWPE and NFSFS's Michell-Kelvin mode were used to investigate SWPE's far field accuracy [6], the investigation of the impact of the various boundary condition approximations was reported separately [13].

Many if not most ships have a transom stern, where the longitudinal slope $Y_{x}$ of the hull becomes large. While this is a violation of the assumptions of thinship theory, it is accommodated numerically by integrating (6) by parts to produce

$$
\begin{aligned}
& P_{\mathrm{T}}^{\mathrm{F}}(\theta)+i Q_{\mathrm{T}}^{\mathrm{F}}(\theta)=\iint_{\mathrm{B}} \mathrm{d} \xi \mathrm{d} \zeta \mathrm{Y}(\xi, \eta, \zeta) e^{i k_{1}(\xi \cos \theta+\eta \sin \theta)+k_{1} \zeta} \\
& -\left.\frac{1}{i k_{1} \cos \theta} e^{i k_{1}(\xi \cos \theta+\eta \sin \theta)} \int d \zeta Y(\xi, \eta, \zeta) e^{k_{1} \zeta}\right|_{\xi=\xi_{\text {bow }}} ^{\xi=\xi_{\text {stern }}} .
\end{aligned}
$$

This form of the free wave spectrum has the additional advantage that it uses the hull offsets directly rather than their $x$ derivatives. Further, the offsets at the bow are typically zero and so contribute nothing to its second term. This formulation was implemented in SWPE v3. 
Also introduced was a viscous damping factor $\exp \left[\left(-2 k_{0}^{2} v x \sec ^{4} \theta\right) / \mathrm{U}\right]$ which, when injected in the integrand of (5), aided the numerical convergence of the process. The factor was revised in SWPE v6.

The aft-of-stern far field calculation was naïvely ${ }^{1}$ complemented by a routine that calculated the far field component between bow and stern using (4) without swapping the order of integration. (Ahead of the bow the far field component is zero.) This component was the most time consuming to calculate due to its computationally expensive triple integral.

Coupled with a local field computation as per (3), we were finally able to compute the total velocity potential everywhere, and that marked the completion of SwPE v3. Figure 1 shows, for a David Taylor Model Basin Model 5415 (length $5.72 \mathrm{~m}$, speed $2.064 \mathrm{~ms}^{-1}$ and acceleration due to gravity $9.81 \mathrm{~ms}^{-2}$ ), the contribution of the local field component to the total wave elevation along the side of the ship at a distance equal to one-tenth its length. Also produced were figures showing the effects of viscous damping, the effects of the transom stern formulation and a comparison with results calculated by others [7].

The general expression for a Havelock source in a fluid of finite depth $h$ [14] is significantly more complex than its infinite depth counterpart but, when evaluated on the free surface $z=0$, its far field component is

$$
\begin{aligned}
& \mathrm{G}^{\mathrm{F}}(x, y, 0 ; \zeta) \\
& =-\frac{k_{0}}{\pi} \mathfrak{R i} \int_{-\pi / 2}^{\pi / 2} \mathrm{~d} \theta \sec ^{2} \theta e^{-i k_{2}(x \cos \theta+y \sin \theta)} \frac{\cosh k_{2}(\zeta+h) \operatorname{sech} k_{2} h}{1-h k_{0} \sec ^{2} \theta \operatorname{sech}^{2} k_{2} h}
\end{aligned}
$$

where $k_{2}$ is given implicitly as the solution of $k_{2}=k_{0} \sec ^{2} \theta \tanh k_{2} h$. Replacing the infinite-depth form of $\mathrm{G}^{\mathrm{F}}$ with this finite-depth form in (4) and swapping the orders of integration as before produces

$$
\phi^{\mathrm{F}}(x, y, 0)=-2 \mathrm{U} \frac{\mathrm{k}_{0}}{\pi} \Re \int_{-\pi / 2}^{\pi / 2} \mathrm{~d} \theta \sec \theta \mathrm{k}_{2} e^{-i k_{2}(x \cos \theta+y \sin \theta)}\left[\mathrm{P}_{\mathrm{T}}^{\mathrm{F}}(\theta)+i \mathrm{Q}_{\mathrm{T}}^{\mathrm{F}}(\theta)\right]
$$

${ }^{1}$ We found later a way to generalise (5) which was then implemented in SwPE v5. 


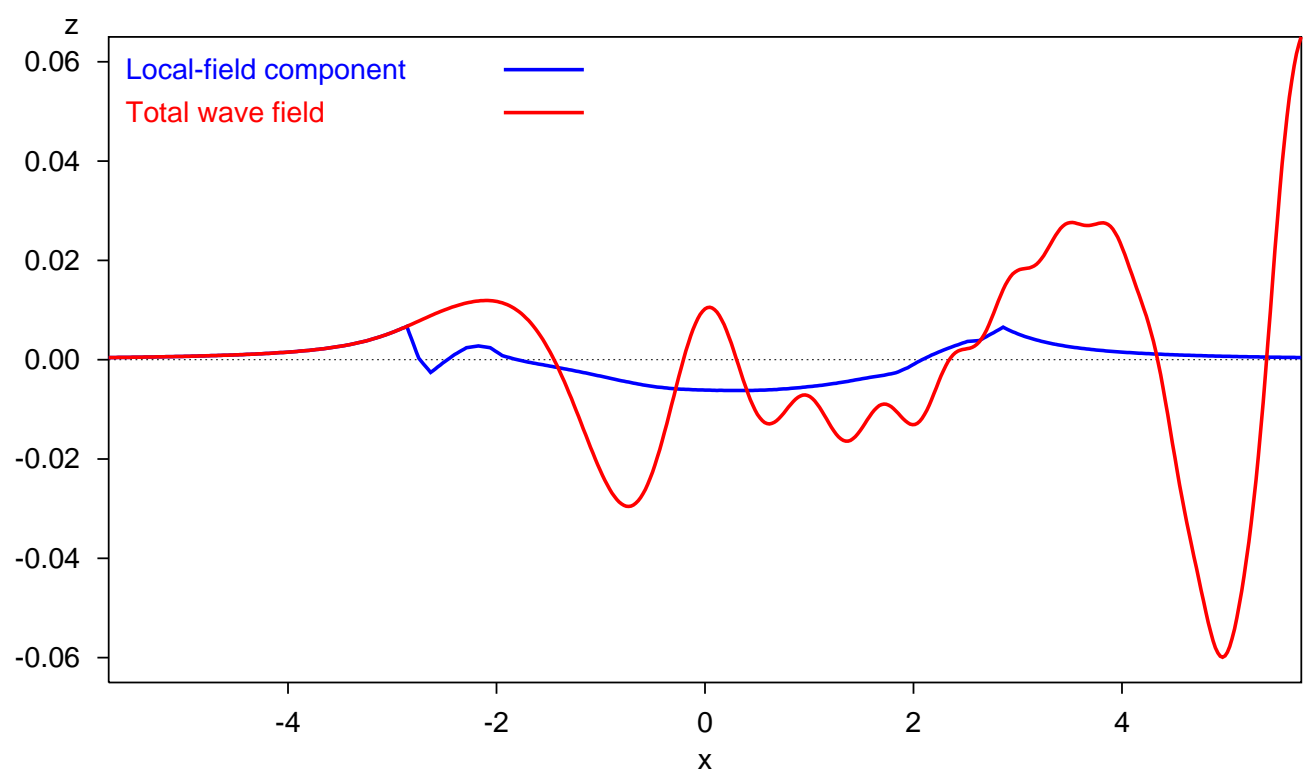

Figure 1: Comparison of the total wave elevation and its local field component along $y=0.572 \mathrm{~m}$ for a Model 5415 hull of length $5.72 \mathrm{~m}$ travelling at speed $2.064 \mathrm{~ms}^{-1}$ with acceleration due to gravity $9.81 \mathrm{~ms}^{-2}$. 
where

$$
\begin{aligned}
& P_{T}^{\mathrm{F}}(\theta)+i Q_{T}^{\mathrm{F}}(\theta) \\
& =\frac{1}{-i k_{2} \cos \theta} \iint_{\mathrm{B}} \mathrm{d} \xi \mathrm{d} \zeta Y_{\xi}(\xi, \eta, \zeta) e^{i k_{2}(\xi \cos \theta+\eta \sin \theta)} \frac{\cosh k_{2}(\zeta+h) \operatorname{sech} k_{2} h}{1-h k_{0} \sec ^{2} \theta \operatorname{sech}^{2} k_{2} h}
\end{aligned}
$$

Unfortunately, no equivalent of Newman's local field polynomial approximations have been developed for finite fluid depth so SWPE v4 is restricted to calculating the far field component only, and even then only for the free surface. Nevertheless, the dramatic impact of finite depth on the wake's envelope can be observed from the far field component alone [8].

SWPE v4 also saw the extension to calculating flows produced by vessels with multiple hulls (for example, catamarans) or, equivalently, multiple vessels. SWPE calculates a potential that is subject to linear boundary conditions. Linear superposition allows perturbation velocity potentials produced by individual hulls to be combined to yield the perturbation velocity potential that would have been produced by all hulls combined. This was easy to implement and allowed for interesting investigations into constructive and destructive interference [8]. Figure 2 shows the combined wave pattern produced by a DDG51 destroyer, a US Los Angeles class submarine and a Wigley-hulled catamaran, each travelling at 30 knots.

Version 5 of SWPE saw some significant improvement in terms of precision, speed and functionality. Precision was improved by using second order integration routines for all integrations over the hull.

A major increase in computational speed was achieved by making an observation (drawn from extending the concept of transom sterns to transom bows and then combining with superposition for multihulls) that two hulls in tandem, with the transom stern of the first butted against the transom bow of the second, should produce waves as though they were one hull (which, of course, they are!). Their combined far field components at their intersection would be due entirely to the first hull because the second has no far field 


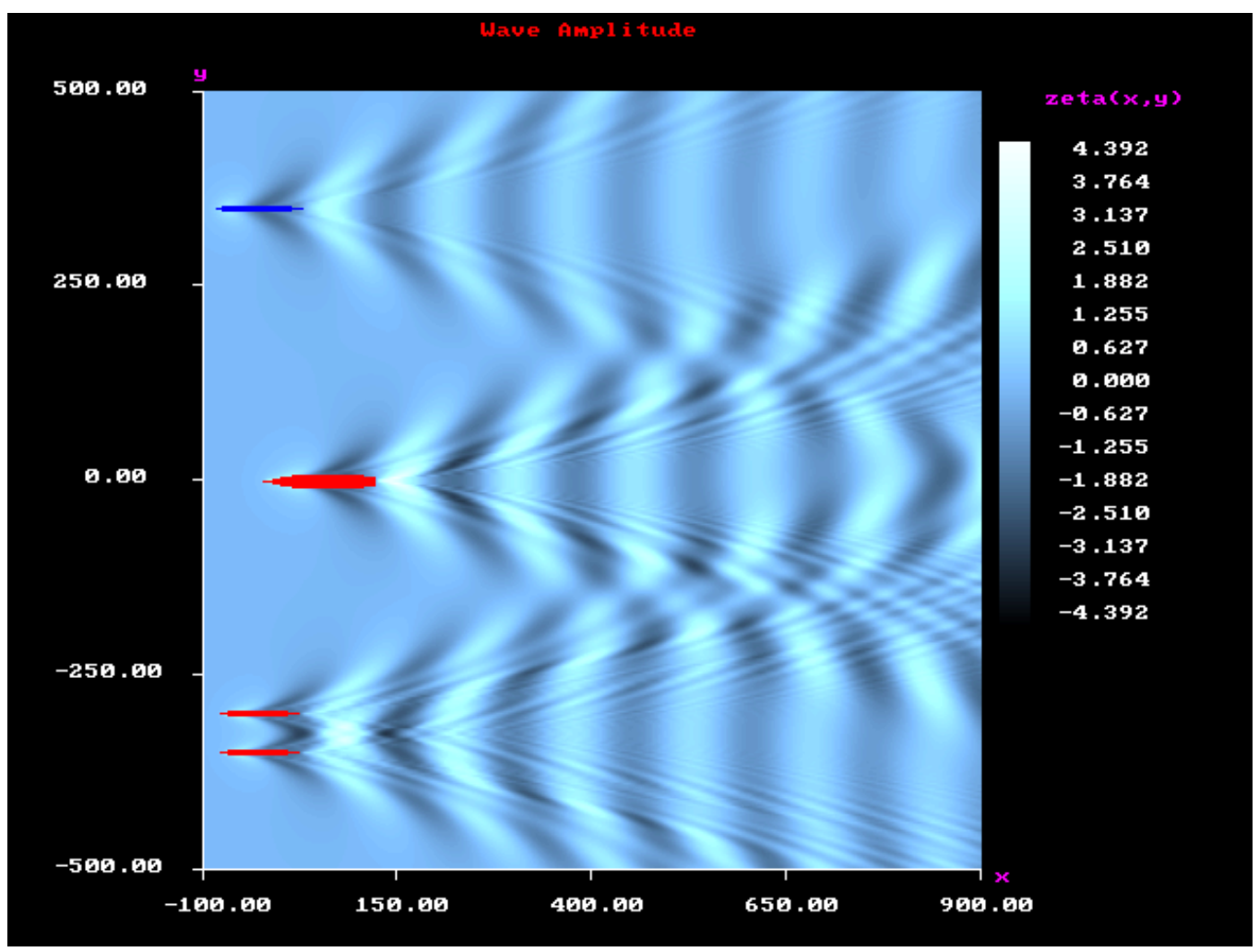

Figure 2: Combined wave pattern produced by a DDG51 destroyer, a US Los Angeles class submarine and a Wigley-hulled catamaran, each travelling at 30 knots. The submarine (uppermost vessel) is only just submerged. 
component ahead of its bow. That is, part way along a ship's hull the far field component is due only to the part of the hull that lies ahead. This led to the idea that it must be possible to calculate the far field component at $(x, y, z)$ between the bow and stern by integrating not over the entire body but only from bow to $x$.

Recall that (2) applies only for $x>0$ and that $G^{F}=0$ for $x<0$. This can be formalised by introducing the Heaviside step function $\mathrm{H}(\mathrm{x})$ into (2) to give

$$
\mathrm{G}^{\mathrm{F}}(x, y, z ; \zeta)=-\frac{k_{0}}{\pi} H(x) \Re i \int_{-\pi / 2}^{\pi / 2} d \theta \sec ^{2} \theta e^{-i k_{1}(x \cos \theta+y \sin \theta)+k_{1}(z+\zeta)}
$$

from where it follows that $\phi^{\mathrm{F}}(x, y, z)$ is as before but with

$$
P_{\mathrm{T}}^{\mathrm{F}}(\theta)+i Q_{\mathrm{T}}^{\mathrm{F}}(\theta)=\frac{1}{-i k_{1} \cos \theta} \iint_{\mathrm{B}} \mathrm{d} \xi \mathrm{d} \zeta Y_{\xi}(\xi, \eta, \zeta) e^{i k_{1}(\xi \cos \theta+\eta \sin \theta)+k_{1} \zeta} \mathrm{H}(x-\xi) .
$$

Effectively, this is an integration not over the whole of the hull but only over the portion that lies ahead of the field point of interest $(x, y, z)$.

Modification to the program allowed the far field component to be calculated everywhere using the same technique that had previously only been available aft of the stern. It increased the speed of calculation of the far field component between bow and stern by a factor of around 50 (more specifically, a factor equivalent to the number of columns of field points). Previously that component had been the most time consuming to calculate, so the increase in computation of this component translated to a significant increase in the overall speed of the program [9].

In a similar way the functionality of the program was increased by allowing the submergence parameter $\mathrm{H}$ to take on a negative value but for the body integration to be done only for the portion of the hull that then lies beneath the free surface. This allowed for hulls to be raised above the free surface and for only the portion that was wet to contribute to the wavemaking. This was used immediately to increase or decrease the draft of ships arbitrarily, without the need for supplying recalculated offsets as input as had been required 
previously. A delightful set of figures of the waves created by a submarine at a range of depths and as it breaches the surface were produced [11].

From there, a natural extension was to calculate the hydrodynamic and gravitational forces and moments acting on the hull and to adjust sinkage and trim until they were in balance. Trim was achieved by a pseudo-rotation, in which each station was raised/lowered by an amount proportional to its distance along the hull. This approximation is acceptable for the small angles of rotation that are typical for a ship's trim. The hydrodynamic forces and moments were calculated from the velocity components over the hull. Sinkage and trim were then solved for in a pair of simultaneous linear equations that balance the (linearised) vertical forces and first moments. This process could be iterated until a desired level of satisfaction had been achieved.

By these enhancements, SWPE v5 was able to represent the hull more accurately, calculate the entire flow significantly faster than previously, adjust the depth or draft of vessels arbitrarily, and balance forces through sinkage and trim. Figures showing comparisons between experimental results and SWPE's calculations of sinkage and trim were also produced [10].

SWPE v6 investigated various alternative formulations for the viscous damping factor. The original formulation introduced in SWPE v3 could be challenged on a number of grounds including dependence on the location of the origin, independence of $y$, violation of Laplace's equation and in particular a lack of memory of the wave's propagation. Various alternative formulations were developed to overcome these objections [12]. The preferred factor, $\exp \left[-4 k_{1}^{2} v(x+y \tan \theta) / u\right]$, still suffers from most of the original objections.

The last version of SWPE, version 7, contained enhancements to calculate wave resistance and viscous resistance.

Although not the subject of the current article, a branch of SWPE, SWPE-PD, was developed to calculate flows due to pressure distributions (for example, hovercraft). Its mathematical formulation has strong similarities to thin 
ship theory [4]. Development was initiated on a further branch, SWPE-FS, intended to calculate flows due to flat ships (for example, speedboats) in which the surfaces due to constant pressure panels (as calculated by SWPEPD) are used to build a system of linear equations for pressures that deform the surface in a way that matches the flat hull shape. Progress on SWPE-FS has been both difficult and limited due to the ill-conditioned nature of the inverse problem that it seeks to solve.

\section{Conclusion}

The Sea Wave Pattern Evaluation (SWPE) suite of programs started out with the modest ambition of calculating far field wave elevations for thin ships but grew to encompass a raft of other tasks including calculating the complete wave field with velocity components for multihull and submerged vessels, incorporating sinkage and trim, viscous damping and finite depth effects, and measuring wave resistance and viscous resistance. It also led to investigations into waves produced by pressure distributions and planing surfaces. This article traces its development, explains the mechanics of its key components, and refers to some of the results that have been produced by it.

Acknowledgments This article is based on work completed jointly with E. O. Tuck and L. Lazauskas. It is the author's firm opinion that no individual or pair from this trio would have been able to produce a result of similar quality without the valuable contribution of the other(s). DSTO provided funding. 


\section{References}

[1] Michell, J. H. The wave resistance of a ship, Phil Mag (5), Vol 45 (1898) 106-123. C318

[2] Newman, J. N. Evaluation of the wave-resistance Green function: Part 1 — The double integral J. Ship Res. 31 (1987) 79-90. C319

[3] Scullen, D. C. Accurate computation of nonlinear free-surface flows, PhD Thesis, The University of Adelaide (1998). http://www.scullen.com.au C321

[4] Scullen, D. C. and Tuck, E. O. Sea wave pattern evaluation - pressure distributions: Mathematical formulation, Scullen \& Tuck Pty Ltd (2001). http://www.scullen.com.au C329

[5] Tuck, E. O., Lazauskas, L. and Scullen, D. C. Sea Wave Pattern Evaluation, Part 1 report: Primary code and test results (surface vessels), Department of Applied Mathematics, The University of Adelaide, April 1999. http://www.scullen.com.au C317, C321

[6] Tuck, E. O., Scullen, D. C. and Lazauskas, L. Sea Wave Pattern Evaluation, Part 2 report: Investigation of accuracy, Department of Applied Mathematics, The University of Adelaide, May 1999. http://www.scullen.com.au C317, C322

[7] Tuck, E. O., Scullen, D. C. and Lazauskas, L. Sea Wave Pattern Evaluation, Part 3 report: Near-Field Waves, Scullen \& Tuck Pty Ltd, Adelaide, January 2000. http://www.scullen.com.au C317, C323

[8] Tuck, E. O., Scullen, D. C. and Lazauskas, L. Sea Wave Pattern Evaluation, Part 4 report: Extension to Multihulls and Finite Depth, Scullen \& Tuck Pty Ltd, Adelaide, June 2000. http://www.scullen.com.au C317, C325 
[9] Tuck, E. O., Scullen, D. C. and Lazauskas, L. Sea Wave Pattern Evaluation, Part 5 progress report: Speed enhancements, Scullen \& Tuck Pty Ltd (2000). http://www.scullen.com.au C317, C327

[10] Tuck, E. O., Scullen, D. C. and Lazauskas, L., Sea wave pattern evaluation Part 5 report: Speed-up and Squat, Scullen \& Tuck Pty Ltd (2001). http://www.scullen.com.au C317, C328

[11] Tuck, E. O., Scullen, D. C. and Lazauskas, L. Sea wave pattern evaluation Submarine portfolio - 10 knots, Scullen \& Tuck Pty Ltd (2000). http://www.scullen.com.au C317, C328

[12] Tuck, E. O., Scullen, D. C. and Lazauskas, L. Sea wave pattern evaluation Part 6 report: Viscosity Factors, Scullen \& Tuck Pty Ltd (2002). C317, C328

[13] Tuck, E. O. and Scullen, D. C. A comparison of linear and nonlinear computations of waves made by slender submerged bodies, Journal of Engineering Mathematics 42 (2002), 255-264. C322

[14] Wehausen, J. H. and Laitone, E. H., Surface Waves, in Handbuch der Physik, ed. Flugge W., Ch. 9. Springer-Verlag, 1962. http://coe.berkeley.edu/SurfaceWaves/ C318, C323

\section{Author address}

1. D. C. Scullen, Scullen \& Tuck Pty Ltd and The University of Adelaide, South Australia, Australia. mailto:david.scullen@scullen.com.au 